\title{
A FMRP-USP em Atividades de Ensino, Pes- quisa e Extensão de Serviços à Comunidade, Dentro do Projeto Centro de Saúde-Escola
}

\author{
Antonio R. Franco'; Nagib Haddad²; Aldaisa C. Forster ${ }^{3}$ \& Maria do Carmo G. Caccia-Bava ${ }^{4}$
}

${ }^{1}$ Docente e Diretor Técnico do CSE. ${ }^{2}$ Docente. Aposentado. ${ }^{3}$ Docente e ex- Diretora Técnica do CSE. Departamento de Medicina Social da Faculdade de Medicina de Ribeirão Preto-USP ${ }^{4}$ Diretora Acadêmica, de Ensino e Pesquisa do CSE-FMRP-USP

Correspondência: Rua Guaritá, 325 - Jd. Recreio. Ribeirão Preto- SP .- CEP: 14040-350 e.mail: gcbava@keynet.com.br

\section{RESUMO HISTÓRICO}

E m 26 de dezembro de 1951, o então governador do Estado de São Paulo, Sr. Lucas Nogueira Garcez, promulga a lei $\mathrm{n}^{\mathrm{o}} 1467$, dispondo sobre organização e finalidades da Faculdade de Medicina de Ribeirão Preto da Universidade de São Paulo ${ }^{(1)}$.

Em seu artigo primeiro, a lei dizia que a faculdade tinha por fim ministrar, desenvolver e aperfeiçoar o ensino e o estudo das Ciências Médicas, em curso de 6 anos, e listava as várias disciplinas, dentre elas a de Higiene. No artigo segundo, citava a constituição das várias cadeiras e departamentos, dentre estes o de Higiene ${ }^{(2)}$.

Vale aqui ressaltar que os legisladores da época já sentiam a importância das atividades de ensino médico junto à comunidade, ao inserirem, no artigo 14 da lei, o seguinte trecho, que, pelo seu valor histórico, deve ser transcrito integralmente:

ARTIGO 14 - Fica criado o Centro de Saúde, anexo à Faculdade de Medicina de Ribeirão Preto, dirigido por um Diretor e orientado por um Conselho, do qual farão parte os professores das cadeiras de Higiene e de Clínica Médica, o Diretor Geral do Departamento de Saúde ou seu delegado, o Diretor do Hospital das Clínicas e o Delegado Regional de Saúde.
Parágrafo $1^{o}$ - Serão estabelecidos no Regulamento da Faculdade os serviços com que contará o Centro de Saúde, as cadeiras a que ficarão subordinados e o entrosamento deles com o Hospital das Clínicas.

Parágrafo $2^{\circ}$ - A área de atuação do Centro de Saúde será estabelecida por entendimento com a Secretaria da Saúde Pública e da Assistência Social."

Entrando essa lei em vigor, em $1^{\circ}$ de janeiro de 1952, foram tomadas providências para instalação da faculdade, iniciando-se, naquele mesmo ano, o curso para a primeira série do ensino médico, com as disciplinas básicas. Entretanto, enquanto a Faculdade de Medicina tomava corpo e seguia sua trajetória, a criação do Centro de Saúde-Escola foi postergada por um bom período de tempo, e isso tem uma explicação.

Em 18 de agosto de 1954, o Prof. Pedreira de Freitas foi contratado como Professor Catedrático de Higiene da Faculdade de Medicina de Ribeirão Preto da USP, tendo larga experiência em pesquisas de campo, principalmente a respeito da epidemiologia da Malária e da Moléstia de Chagas. Era um homem determinado e de firmes convicções a respeito de pesquisa, do ensino da Medicina e, particularmente, do ensino da Medicina Preventiva daquela época. 
De acordo com a lei que criou a FMRP-USP, o curso de Higiene deveria ser dado em apenas um semestre, para alunos do $6^{\circ}$. ano médico. Entretanto, o Professor Pedreira estava convencido da necessidade de que os aspectos preventivos e sociais da Medicina deveriam ser apresentados aos alunos, não como uma especialidade, mas como um ponto de vista, constituindo parte integrante de sua formação. Denominando o novo setor de ensino e pesquisa como Departamento de Higiene e Medicina Preventiva (DHMP), o Professor Pedreira propôs alterações na planificação do Curso de Higiene, sendo as principais as que vêm a seguir:

$1^{\mathrm{a}}$ - A necessidade de ensinar Higiene e Medicina Preventiva não como uma disciplina isolada, "mas como um ponto de vista que devia permear a formação do futuro médico", principalmente na sua atividade junto ao paciente. Tinha convicção de que era absolutamente necessário fazer o ensino da cadeira, encarada, antes, como Medicina Preventiva, junto ao setor clínico e em estreita colaboração com outras cadeiras.

$2^{\text {a }}$ - Constituindo a Epidemiologia o principal elemento para conhecimento da história natural das doenças, para fornecer as bases para sua prevenção, reconheceu a importância de contar, no DHMP , com um estatístico. Teve aí início a Disciplina de Matemática Aplicada à Biologia, um diferencial da faculdade em relação às demais do País, o que persiste até os dias de hoje. Para implementar essa nova área de ensino, foi convidado o Prof. Dr. Geraldo Garcia Duarte, que acumulava uma experiência de 10 anos, trabalhando no Departamento de Estatística da Faculdade de Higiene e Saúde Pública da USP.

As convicções que o Prof. Pedreira de Freitas tinha a respeito de como deveria ser focalizado o ensino de Higiene e Medicina Preventiva fortaleceu-se através de duas oportunidades: a primeira, com bolsa da Fundação Rockefeller, em viagem de visitas a vários departamentos de Medicina Preventiva dos Estados Unidos e Porto Rico e a segunda, sua participação oficial no I ${ }^{\circ}$ Seminário sobre o Ensino de Medicina Preventiva, realizado em Viña del Mar, Chile, em outubro de 1955, patrocinado pela Organização Mundial da Saúde e pela Organização Pan-Americana da Saúde.

De acordo com a primeira proposição, exposta acima, o DHMP deveria ser instalado junto às demais cadeiras clínicas, isto é, no Hospital das Clínicas, onde seria ministrado o ciclo de aplicação do ensino médico. O DHMP foi, então, instalado em duas salas cedidas pelo Departamento de Parasitologia. Posteriormente, havendo necessidade de ampliação, transferiu-se para um número maior de salas cedidas pelo Departamento de Microbiologia e Imunologia, também localizado no prédio central da Faculdade de Medicina.

Até 1955, o DHMP contava apenas com 2 docentes, o Prof. Pedreira de Freitas e o Prof. Duarte. Naquele ano, foi contratado o $3^{\circ}$. docente, Professor Dr. Nagib Haddad, que era, por gosto, assíduo estagiário e freqüentador dos cursos de especialização e de aperfeiçoamento da Escola de Saúde Pública da Universidade de São Paulo. Tinha concluído o Curso Normal de Higiene e Saúde Pública, quando aprofundou seus conhecimentos em Bioestatística, Epidemiologia, Administração Geral, Administração Sanitária e em outras importantes disciplinas concernentes ao campo da Saúde Pública.

Se o Departamento de Higiene e Medicina Preventiva deveria ser instalado junto dos demais departamentos clínicos para uma melhor integração entre eles e ensino, como foi, as atividades docentes assistenciais não deveriam se restringir ao interior do Hospital das Clínicas. Seguindo essa filosofia, em 1956, o Departamento de Higiene e Medicina Preventiva e o de Psicologia Médica, então dirigido pelo Prof. Dr. Sergio Rodrigues, e as cadeiras de Psicologia e de Sociologia da Escola de Enfermagem de Ribeirão Preto - USP elaboraram, em conjunto, um programa denominado: Prática Clínica Social, e os alunos do $3^{\circ}$. ano médico visitavam, individualmente, famílias residentes em bairros periféricos da cidade de Ribeirão Preto, a fim de conhecerem os problemas ligados ao modo de viver dessas famílias e identificarem os fatores que pudessem afetar a saúde mental.

$\mathrm{Na}$ busca da ampliação dessa integração das atividades extramuros, em 1956, a par de atividades de ensino teórico e prático de Epidemiologia e Profilaxia e Bioestatística, foi organizado e instalado, no porão de um prédio alugado, situado à Rua Visconde de Inhaúma ${ }^{\circ} 757$, o Programa de Assistência Médica Preventiva e Social a Famílias. Esse programa teve a colaboração, inicialmente, da enfermeira de saúde pública, D. Maria de Lourdes Valada, e, posteriormente, de D. Judith Costa, da Escola de Enfermagem de Ribeirão Preto. A Professora Judith tinha grande experiência vivida em trabalho no Serviço Especial de Saúde Pública, no centro de saúde de Colatina, Espírito Santo. 
Na parte superior daquele prédio, funcionava o ambulatório de Pediatria e Puericultura, sob a direção do Professor Doutor Jacob Renato Woiski. As crianças atendidas no ambulatório, que necessitassem de visitação domiciliária, eram, então, encaminhadas com seus acompanhantes para inscrição no referido programa.

Todas as pessoas residentes nas casas visitadas eram convidadas a comparecer a esse ambulatório, que tinha por objetivo exercer a Medicina Preventiva nos seus níveis primário e secundário, procurando realizar promoção da saúde, proteção específica, diagnóstico e tratamento precoce de doenças. Havia uma ficha padronizada onde, a par dos dados de identificação pessoal, tais como: procedência, antecedentes familiares e pessoais, condições de trabalho, de moradia, hábitos pessoais, situação vacinal, queixa principal e história da moléstia atual, havia, ainda, espaço para registro dos dados do exame físico geral e do exame físico sistemático dos diversos aparelhos. Eram pedidos rotineiramente: exame de urina tipo I, exame parasitológico de fezes e exame sorológico, para diagnóstico de Sífilis e Doença de Chagas. Os exames eram realizados no Departamento de Parasitologia da FMRP. Os casos mais simples eram tratados e seguidos nesse ambulatório e os que necessitassem de tratamento e acompanhamento especializados eram encaminhados aos departamentos clínicos e cirúrgicos da FMRP.

O Programa de Assistência Médica Preventiva e Social a Famílias serviu não só para o ensino e de local de estágios para alunos de Medicina e de Enfermagem, mas, também, para a realização de inúmeras pesquisas. Solidificava-se, assim, a convicção de que aquela propositura da criação de um Centro de Saúde próprio, por enquanto, era dispensável. Segundo palavras do Professor Pedreira de Freitas,

"O trabalho na comunidade visa fundamentalmente dar ao aluno oportunidade de tomar contato com pacientes no ambiente familiar, de maneira a poderem investigar os fatores físicos, sociais ou mentais que possam, por um lado, estar contribuindo para a doença em causa, por outro lado analisar de que forma essa doença está se refletindo no ambiente social ou familiar. Na mesma oportunidade, o aluno deverá exercer a prevenção em todos os seus níveis, não somente no que diz respeito ao caso em apreço, como também em relação aos demais membros da família. Usará também essa oportunidade para, através de contatos que deva estabelecer para a solução dos problemas que se apresentem, tomar contato com as instituições médico-assistenciais da comunidade, em particular com os serviços oficiais de saúde, particularmente ao nível local."(3)

A partir dessa época, mais entrosado com os departamentos clínicos, esse programa passou a receber, para visitação e acompanhamento, também famílias de pacientes de enfermarias e ambulatórios de outros departamentos.

Foi feito ainda, um acordo com o Dispensário de Tuberculose, pelo qual alguns pacientes, residentes em Ribeirão Preto, eram seguidos pelo DHMP, para tratamento e visitação domiciliária, com a participação de alunos. Outro acordo foi estabelecido com o Centro de Saúde I, da Rua Minas, próximo ao Bosque Municipal, para que alguns pacientes portadores de doenças infecciosas, notificadas, fossem visitados e seguidos por docentes do DHMP, acompanhados de alunos do $4^{\circ}$ ano médico.

Como se poderia prever, a partir de 1961, todas as atividades de ensino do DHMP se desenvolviam junto ao Hospital das Clínicas, conforme a proposta inicial do Professor Pedreira de Freitas de integrar o ensino com as várias clínicas e incorporar, no aluno, a atitude preventiva, em seus vários níveis, com atividades na comunidade.

As tentativas de trabalho na comunidade sempre eram feitas na base de acordos, entre departamentos e também com serviços da Rede de Saúde Publica do Estado. Esses acordos eram não muito fáceis de serem cumpridos, dependiam muito do interesse de alguns professores dos vários departamentos, mas estavam dentro da maneira de pensar e agir do Professor Pedreira de Freitas, que a respeito dizia:

"A lei 1467 de criação da Faculdade de Medicina de Ribeirão Preto cuidou especificamente da criação de um Centro de Saúde anexo a essa Faculdade e orientado por um conselho do qual faria parte o Professor de Higiene. Apesar de ardente defensor da importância do trabalho na comunidade para a formação do futuro médico, não estou convencido da necessidade de a FMRP contar para isso com uma área especial de treinamento, como seria um Centro de Saúde sob o seu controle direto. Sou mesmo de opinião que visan- 
do a formação do futuro médico que, particularmente no Brasil, terá que lidar com indivíduos vivendo em comunidades onde, com freqüência, são muito deficientes os serviços de saúde, será preferivel que o estudante tome contato com pacientes em ambiente que não difira daquele que usualmente irá encontrar. Acresce que a não existência de um serviço de saúde organizado, de maneira a solucionar eficientemente os problemas médico-sociais encontrados, deixa ainda mais evidente a necessidade de se recorrer a todas as instituições médicosociais da comunidade para esse fim. Ao lado das vantagens que apresenta o trabalho em uma comunidade não organizada do ponto de vista da saúde pública, resulta que procedendo dessa forma com freqüência problemas médico-sociais encontrados pelos alunos na comunidade não podem ser solucionados. Porém vale a pena chamar a atenção para que ainda aqui há um aspecto positivo para o aprendizado: o estudante tem um exemplo vivo a favor das noções que lhe são apresentadas no ensino teórico a respeito das vantagens de programas do tipo de planos integrados de saúde."(3)

Em suma, o Professor Pedreira de Freitas achava que não seria conveniente a FMRP ter um Centro de Saúde próprio e/ou anexo, o qual certamente teria as melhores condições de organização e funcionamento, polivalente e dinâmico, que serviria como um modelo ideal a ser mostrado aos alunos, quando, na realidade, fora da FMRP, havia uma rede de saúde arcaica, inoperante e sem nenhuma integração entre as unidades especializadas. Ele estava convicto de que o ensino deveria mostrar a realidade da Rede de Saúde Pública, tal como ela estava estruturada, com a qual o aluno iria lidar depois de formado. $\mathrm{Ou}$ seja, um CSE próprio seria uma vitrine destoante da realidade.

O falecimento prematuro do Professor Pedreira de Freitas, ocorrido no ano de 1968, trouxe uma profunda perda para o DHMP, que tinha apenas pouco mais de uma década de existência. Foi uma perda também para ciência e para o ensino médico. Entretanto, os rumos estavam traçados. Foi um pioneiro no Ensino da Medicina, com apurado senso de visão do futuro. Posicionava-se claramente a favor de um ensino fundamentado na integração entre as várias disciplinas, entre os vários departamentos clínicos e, ao mesmo tempo, inserido na comunidade.

\section{A CRIAÇÃO DO CENTRO DE SAÚDE-ES- COLA}

Com o advento da Reforma Sanitária, instituída pelo Professor Leser, já não subsistiam mais as ponderações iniciais do Professor Pedreira de Freitas, pois a estrutura e funcionamento da Rede de Saúde Pública, no Estado de São Paulo, estava sendo completamente reformulada, com a criação de coordenadorias e extinção dos órgãos inoperantes e burocráticos e os centros de saúde tornando-se polivalentes e eficientes.

Com a criação da carreira de sanitarista, na Secretaria de Estado da Saúde, na década de 70, a Residência em Medicina Preventiva, iniciada em 1962, necessitava de uma reformulação, visto que os objetivos primeiros dessa residência eram, até então, mais voltados a uma programação visando ao preparo de um docente de Medicina Preventiva ou ao aperfeiçoamento de um médico que desejasse trabalhar como generalista junto a uma comunidade. Começou-se a discutir, no âmbito do Conselho do Departamento de Medicina Social (nova denominação do antigo Departamento de Higiene e Medicina Preventiva, após a implantação da reforma na Universidade de São Paulo, em 1970), como seria a nova estruturação da Residência em Medicina Preventiva, dada a possibilidade de sua equiparação com o curso de saúde pública, para ingresso na carreira de sanitarista.

Por outro lado, as atividades junto à comunidade urbana da cidade de Ribeirão Preto, para serem implementadas, necessitavam de uma área maior e mais sólida, que não dependesse de acordos frágeis e vulneráveis às injunções e interferências externas, e que atendesse plenamente às necessidades de ensino, não só para os alunos de graduação, como também para os médicos residentes.

Renascia, então, a idéia de se criar um Centro de Saúde-Escola, ligado à Faculdade de Medicina de Ribeirão Preto, resgatando, assim, agora em uma realidade sociopolítica mais oportuna, a proposta contida no artigo 14 da lei $n^{\circ} 1.467$ de 26/12/1951, que criou a FMRP-USP, conforme exposto anteriormente.

É oportuno ressaltar que, já na década de 60 e durante a de 70, no bojo das discussões da Reforma Sanitária, os vários Departamentos de Medicina Preventiva das Escolas Públicas de Medicina do Estado de São Paulo, iniciaram um movimento articulado para definir, dentre outras coisas, os conteúdos programáticos de suas disciplinas, tanto para os cursos de graduação quanto para os de residência em Medicina 
Preventiva. Segundo Yazlle Rocha ${ }^{(4)}$, "esse movimento trazia no seu bojo inovações metodológicas e doutrinárias: incorporando a bioestatística, a metodologia epidemiológica, o modelo da historia natural das doenças e a assistência integral à saú$d e$ ". Continua o autor:

"foi no cenário desse fórum de discussão, que os Departamentos de Medicina Preventiva focalizaram como objeto de estudo e intervenção 'a comunidade'- sem maior clareza e precisão incorporando, assim, um objeto social que, como descobriríamos mais tarde, implica diferentes $e$ antagônicas concepções”.

Como foi dito anteriormente, o cenário sociopolítico era outro e os acontecimentos, tanto intramuros quanto fora, dependiam muito pouco da vontade e de convicções pessoais. A necessidade/disposição de "ir à comunidade", entretanto, é honesto que se diga, não era unanimidade nem dentro dos próprios Departamentos de Medicina Preventiva, muito menos das Escolas Médicas, como, aliás, ainda acontece nos dias atuais. De qualquer forma, percebeu-se que, já que precisavam sair, era de todo impossível e inadequado ministrar as disciplinas desses departamentos, utilizando serviços alheios aos seus interesses de ensino e que não fossem dirigidos por eles próprios, com certa autonomia. Ressalte-se que, nessa altura dos acontecimentos, muitos desses departamentos já contavam com seus Centros Médicos Sociais e Comunitários, mais tarde, Centros de Saúde-Escola.

Dessa maneira, desejando que o Centro de Saúde-Escola da Faculdade de Medicina de Ribeirão Preto fosse um órgão de toda a faculdade e não apenas do Departamento de Medicina Social, o Professor Nagib Haddad, então chefe desse departamento, numa reunião da Comissão Coordenadora de Ensino da Faculdade (CCE), propôs que se formasse uma comissão oficial para visitar os Centros de Saúde-Escola já existentes: da Faculdade de Ciências Médicas da Santa Casa de São Paulo, na Barra Funda; o da Faculdade de Medicina da USP, no Butantã e o da Faculdade de Medicina de Botucatu. Após essas visitas, realizadas no início de 1976, foi rediscutido o assunto no âmbito do Conselho do Departamento de Medicina Social e da Comissão Coordenadora de Ensino da Faculdade ${ }^{(5 / 8)}$.

Com base em uma minuta-padrão, foi, então, elaborada uma proposta de convênio entre a Faculdade de Medicina de Ribeirão Preto e a Secretaria de Estado da Saúde. A minuta definitiva foi encaminha- da para as respectivas aprovações na Comissão Coordenadora de Ensino e na Congregação da Faculdade, tendo sido aprovada em ambas as instâncias, no ano de 1977.

Finalmente, em 28 de dezembro de 1978, o referido convênio foi assinado, chegando à FMRP-USP na segunda quinzena de janeiro de 1979. O Diretor da Faculdade designou o Chefe do Departamento de Medicina Social, Prof. Dr. Nagib Haddad, como seu representante junto ao Conselho Diretor do CSE e o Coordenador de Saúde da Comunidade da Secretaria de Estado da Saúde designou o Dr. Edgard Rolando como seu representante no Conselho Diretor do CSE. O Prof. Dr. Juan Stuardo Yazlle Rocha foi indicado como representante do Departamento de Medicina Social junto ao conselho e o Prof. Dr. Jarbas Leite Nogueira como $1^{\circ}$. Diretor Técnico do Centro de Saúde-Escola de Ribeirão Preto, exercendo seu mandato no biênio 1980/1981.

Até a presente data, muita coisa aconteceu, muitas dificuldades foram superadas, muitos professores revezaram-se na Direção Técnica do CSE, cada um deixando seu legado de trabalho e dedicação, permitindo que a Faculdade de Medicina de Ribeirão Preto da Universidade de São Paulo, nos dias atuais, disponha de um projeto ímpar de ensino, pesquisa e de extensão de serviços à comunidade, que ultrapassa em muito, o que se imaginava no início, um simples Centro de Saúde da Rede Pública de Saúde.

\section{O projeto Centro de Saúde-Escola: o que é e o que pretende ser}

A evolução do Centro de Saúde-Escola, nos 23 anos de existência, tem ensejado muitos momentos de reflexão por parte de todos aqueles que vêm acompanhando sua trajetória repleta de desafios para o processo de formação e capacitação de recursos humanos, produção de conhecimentos e de organização do trabalho de assistência à saúde. Docentes, técnicos, usuários, alunos, pesquisadores, em diferentes momentos, envolveram-se nesse exercício dinâmico de ensinar e aprender.

Esses desafios se fazem sempre presentes, ao considerarmos que tanto a formação/capacitação de recursos humanos, quanto a geração de novos conhecimentos e a opção por modelos assistenciais, envolvem dimensões não apenas técnicas e operacionais, mas, também, valores éticos e políticos, presentes na relação cotidiana com o ensino e com o trabalho desenvolvidos no CSE. 
Através de diferentes estratégias derivadas de momentos e movimentos políticos mais amplos, os objetivos do CSE são, e sempre foram, desde sua fundação, nos idos de 1979, os mencionados a seguir.

1- A formação de alunos de graduação do curso de Ciências Médicas da FMRP, e de novos profissionais de outras unidades, como Enfermagem, Psicologia, Odontologia e Farmácia, oferecendo campo de ensino teórico e prático para o processo de ensino e aprendizagem, sensível às mudanças curriculares pelas quais passam as instituições formadoras.

2- A capacitação de profissionais da Rede Pública de Serviços de Saúde do Município e da Região, tanto de nível médio quanto de universitário e de distintas formações.

3- A assistência aos grupos sociais, presentes na área geográfica de abrangência de suas unidades de saúde: Ipiranga e Vila Tibério, a partir de 1979; Sumarezinho, em 1981; Núcleo de Saúde da Família I, inaugurado em 1999; Núcleo de Saúde da Família II e Núcleo de Saúde Mental, instalados no ano 2000; Núcleos de Saúde da Família Família III, IV e V, iniciados em 2001.

4- A geração de novos conhecimentos e tecnologias para a área de atenção primária, em especial, e da saúde, em geral.

Nesse caminhar, muitos parceiros se agregaram, deixando suas contribuições. Entretanto, esse projeto não teria sido possível sem o Departamento de Medicina Social, que, pioneiramente, desencadeou o processo de constituição do CSE, administrou-o generosamente, abrindo espaços, para o ensino, para tantos outros departamentos da própria faculdade, como o de Ginecologia e Obstetrícia, em 1981; Pediatria, em 1991; Clínica Médica, em 2000; Neurologia, Psiquiatria e Psicologia Médica e Oftalmologia, em 2000, e Ortopedia, em 2002.

Quanto a outras unidades do Campus USP, abriu-se para a Escola de Enfermagem, desde 1983, e, após período de relações mais esparsas, ela inseriuse no CSE como parceira solidária no projeto de criação dos Núcleos de Saúde da Família III, IV e V; para o Departamento de Psicologia da Faculdade de Filosofia, Ciência e Letras, desde 1986; para a Faculdade de Odontologia, em 1987, e com o retorno formal no ano 2000, e, ainda, para a Faculdade de Ciências Farmacêuticas, que, embora estivesse sempre presente, desde o ano de 1992, intensificou suas relações a partir de 2000, trazendo novos recursos, do- centes, alunos e pesquisadores, com significativa presença no Núcleo de Saúde da Família III.

No presente momento, em que se ampliam as atividades universitárias dentro do espaço do CSE, desencadeia-se o processo de formalização das suas relações com o Hospital das Clínicas, tornando-o de fato e de direito signatário do convênio, que ora se reformula entre a Universidade de São Paulo e a Prefeitura Municipal de Ribeirão Preto, fazendo cumprir, enfim, o que previa a lei inicial de criação da faculdade, embora, a parceria de fato entre as duas instituições acompanhe a própria história do CSE.

No que tange às atividades de assistência, o Centro de Saúde-Escola, no momento de sua inauguração, tinha como parâmetro de referência os programas definidos pela Secretaria Estadual da Saúde, voltados para as áreas da criança e da gestante, e o Programa Nacional de Imunização. Essas ações eram desenvolvidas, inicialmente, em suas duas unidades pioneiras, Ipiranga e Vila Tibério, sendo que, na primeira, havia ainda os Sub-programas de Controle da Hanseníase e da Tuberculose, e, na segunda, o atendimento odontológico.

Em 1981, inaugurou-se a Unidade CSE Sumarezinho, que começou a operar efetivamente em 1982. A atenção ao adulto foi deslocada para tal unidade, ficando as duas primeiras exclusivamente como unidades maternoinfantis. Nela, gradativamente, foram estruturados outros serviços, como o de Ginecologia e Obstetrícia, em 1982, Pediatria, Serviço Social e Psiquiatria, em 1983; Psicologia e Oftalmologia, em 1984; Cardiologia, em 1987; Ambulatório de AIDS, em 1998, e Saúde do Trabalhador, em 1989.

Ao corpo técnico inicial, em sua maioria vinculado à Secretaria Estadual da Saúde, foram somandose outros profissionais, chegando-se a 76 técnicos de níveis médio, básico e universitário, contratados pelo convênio entre a Universidade de São Paulo e a SES. Assim, a capacidade assistencial pôde expandir-se e estimular a presença de novos departamentos da FMRP e outras unidades do Campus, com seus alunos, residentes e pós-graduandos, além de pesquisadores com diversos vínculos, entusiasmados pelas novas possibilidades de produção do conhecimento, trazidas pela crescente diversificação temática, apresentada pelo CSE.

Se, em todo o ano de 1979, o CSE realizou perto de 12.000 consultas médicas, em 1989, esse número chegava perto de 60.000 . Com a chegada de novas especialidades, com a alocação de 145 profissionais 
da Secretaria Municipal de Saúde para a abertura do Pronto-Atendimento em regime de 24 h para a Clínica de adultos, em 1993, e 24 h para a Pediatria, em 1997, as 12.000 consultas/ano de todo o CSE transformaram-se rapidamente em 12.000 consultas/mês, apenas na unidade-sede.

Quanto aos trabalhos de investigação, lá realizados, a trajetória foi semelhante: de um número pouco expressivo existente no começo da década de 80 , com perto de 36 trabalhos, passou a gerar cerca de 10 a 12 novos projetos/ano, de 90 a 95, subindo para uma média de 24 trabalhos/ano na segunda metade da década de 90 . Ao atingir o ano de 2000, com o processo de criação dos Núcleos de Saúde da Família tivemos 33 trabalhos em 2000, 32, em 2002, e 57 novos projetos de janeiro a junho de 2002, em sua expressiva maioria, abordando a temática do atendimento básico à saúde, e, mais especificamente, o Programa de Saúde da Família.

Esse incremento de projetos de investigação, existente em todas as unidades do CSE, levou à instalação de um comitê próprio, o Comitê de Ética em Pesquisa, ocorrida no ano de 2000, operando ativamente desde então, com o reconhecimento do CONEP.

$\mathrm{O}$ crescimento da presença acadêmica em suas unidades, também bastante expressivo, vem desenvolvendo-se no contex to da política municipal de saúde, que, pela diretriz de hierarquização da assistência à saúde, esvazia as possibilidades de aprendizado no hospital universitário, para agravos primários e secundários à saúde.

Assim, o CSE recebe, hoje, cerca de 1000 diferentes alunos de graduação/ano, matriculados em disciplinas regulares, desenvolvidas pela Faculdade de Medicina, Escola de Enfermagem, Faculdade de Farmácia, Faculdade de Odontologia, e curso de Psicologia, além de alunos vinculados a Projetos Bolsa-Trabalho.

No nível de pós-graduação lato sensu, vem acolhendo Programas de Residência Médica nas áreas de Ginecologia e Obstetrícia ( $2^{\circ}$ e $3^{\circ}$ anos); Pediatria e Neuropediatria; Clínica Médica, Pneumologia e Moléstias Infectocontagiosas, Geriatria, Medicina Geral e Comunitária, com Ênfase em Saúde da Família, na $4^{\mathrm{a}}$ turma. No ano de 2002, amplia-se ainda mais, com a abertura do Serviço de Ortopedia, estruturando campo de formação para o Programa de Residência Médica em tal especialidade.

Para além do horizonte acadêmico, o CSE é também campo de estágio fundamental para o $\mathrm{I}^{\circ} \mathrm{Cur}-$ so de Especialização em Saúde da Família, promovido pelo Pólo Norte/Oeste Paulista de Formação Acadêmica e Capacitação de Recursos Humanos para a Saúde da Família, ministrado através da Faculdade de Medicina e da Escola de Enfermagem e destinado a médicos, enfermeiros e dentistas inseridos em equipes do PSF de Ribeirão Preto e de mais 16 municípios da Região.

Para o nível médio de ensino, acolhe cerca de 70 alunos/ano dos cursos de Auxiliar e Técnico de Enfermagem do Centro Interescolar do Hospital das Clínicas da FMRP-USP.

Embora, a partir de 1998, em significativo momento de sua trajetória, o CSE tenha sido guindado a um novo status institucional, vinculando-se diretamente à Diretoria da FMRP, indicando o reconhecimento de sua importância para a viabilização da política institucional da FMRP, novas e antigas questões ainda carecem de reflexão aprofundada, como as mencionadas a seguir.

a- A convivência entre ensino e prestação direta da assistência, com seus campos de aproximação e também de conflitos, é, certamente, uma delas. Muito tem sido falado do diferente timing da instituição formadora, mais lenta e estável, e da instituição prestadora, mais premida por cobranças políticas, quanto aos impactos gerados.

b- As potencialidades e limites da interdisciplinaridade e do trabalho multiprofissional, quando convivem saberes e profissões cunhados no espírito corporativo, mas, muitas vezes, dispostos e estimulados a revalorizar a vida e a humanizar suas práticas.

Embora muito já tenha sido construído e alcançado, novos desafios se colocam a cada dia, exigindo investimento constante na preparação da equipe, para um intenso trabalho multiprofissional, interdisciplinar e interinstitucional, comprometido com o projeto comum de assistir, formar e gerar conhecimentos com excelência, para o qual o CSE apresenta um potencial ímpar. Pretende, assim, consolidar-se ser um centro universitário sólido e amplo, que permita à Faculdade de Medicina de Ribeirão Preto da Universidade de São Paulo cumprir seu destino, traçado por seus pioneiros, na busca incessante da formação de profissionais da saúde das mais variadas especialidades, tecnicamente competentes e politicamente sensíveis para compreender as reais necessidades do nosso País, como preconizava Pedreira de Freitas. 


\section{REFERÊNCIAS BIBLIOGRÁFICAS}

1 - SÃO PAULO (ESTADO). Lei $\mathrm{n}^{\circ} 161$ de 24 de setembro de 1948. Dispõe sobre a criação de estabelecimentos de ensino superior em cidades do interior do Estado e dá outras providências. Diário Oficial do Estado, São Paulo, 26 set. 1948.

2 - SÃO PAULO (ESTADO). Lei ํㅜ․ 167 de 26 de dezembro de 1951. Dispõe sobre a organização e finalidades da Faculdade de Medicina de Ribeirão Preto da Universidade de Sâo Paulo. Diário Oficial do Estado, São Paulo, 28 dez. 1951.

3 - PEDREIRA DE FREITA JL. Memorial. Apresentado à Comissão Julgadora do Concurso para provimento efetivo do cargo de Professor Catedrático da Cadeira ${ }^{\circ} 10$ - Higiene e Medicina Preventiva e Estatística. Faculdade de Medicina de Ribeirão Preto da USP, Ribeirão Preto, 1963.

4 - YAZLLE ROCHA JS. A Medicina Preventiva - Idéias e práxis. Apresentação. In: Saúde da comunidade. Faculdade de Ciências Médicas de Botucatu da UNESP-Editora, Botucatu (Prelo).
5 - UNIVERSIDADE DE SÃO PAULO. FACULDADE DE MEDICINA DE RIBEIRÃO PRETO. Ata da 330a. Sessão da Congregação, realizada em 14 set. 1979. Ribeirão Preto, 1979.

6 - UNIVERSIDADE DE SÃO PAULO. FACULDADE DE MEDICINA DE RIBEIRÃO PRETO. Ata da 332a. Sessão da Congregação, realizada em 12 out. 1979. Ribeirão Preto, 1979.

7 - UNIVERSIDADE DE SÃO PAULO. FACULDADE DE MEDICINA DE RIBEIRÃO PRETO. Ata da 351a. Sessão da Congregação, realizada em 15 ago. 1980. Ribeirão Preto, 1980.

8 - UNIVERSIDADE DE SÃO PAULO. FACULDADE DE MEDICINA DE RIBEIRÃO PRETO. Ata da 353a. Sessão da Congregação, realizada em 12 set. 1980. Ribeirão Preto, 1980. 